\section{Aspectos estéticos de la interacción}

\author{
Umberto Roncoroni
}

A pesar de que la tecnología digital más o menos inteligentes los entornos profesionales y familiares de las sociedades globalizadas, no observamos, en el plano de la cultura, aquellos cambios que semejante invasión debería implicar. En comparación con la fotografía o el cinematógrafo, que desde sus inicios plantearon cuestiones revolucionarias, la tecnología digital parece actuar solamente en el nivel cuantitativo (más velocidad, más información, etcétera), pero sin tocar las estructuras profundas de la creatividad y de la comunicación. Ahora, esta debilidad que los utopistas de la cybercultura no siempre reconocen, se hace emblemática en la práctica artística: luego de 40 años de experimentos, la computadora no tiene todavía una estética y un lenguaje expresivo propios y comparables con los que los medios de la reproducibilidad técnica supieron a su tiempo desarrollar y lograr. Desde mi punto de vista hay dos razones para esto: una filosófica y una tecnológica. En primer lugar, la misma idea de un pensamiento estético fuerte pierde sentido en esta época posmoderna, caracterizada por la indeterminación, la fragmentación y la deconstrucción de los grandes relatos. En segundo lugar, tenemos en la tecnología digital y en la computadora un sistema complejo que no se puede comparar, así de simple, con la naturaleza y los procesos de los medios tecnológicos analógicos, como la fotografía o la televisión. 
Pero estos problemas están directamente relacionados: por un lado la tecnología se utiliza de modo obsoleto por falta de una estética, y por el otro no se desarrolla una estética porque se aplica mal la tecnología. Sucede, pues, que con los software comerciales se utiliza la computadora como si fuera, según el caso, una cámara fotográfica, la paleta de un pintor, un instrumento musical, una máquina para escribir o - lo que no cambia los términos del problema - una mezcla de estas herramientas analógicas. Y también parece que a menudo se olvida que la computadora no es una herramienta ni un medio, sino un sistema lingüístico complejo cuyas identidades culturales, además de los usuarios, incluyen el software y las interfaces. Recientemente, la teórica alemana Inke Arns ha dicho que:

Hoy, en una época en la cual todo nuestro entorno se mediatiza y digitaliza progresivamente y por lo tanto sus procesos se fundamentan cada vez más en el software, es siempre más importante ser conscientes de que el código o el software afectan directamente el espacio real y virtual en el cual nos movemos, comunicamos y vivimos. El software tiene la capacidad directa de desarrollar o inhibir a sus usuarios. ${ }^{1}$
Así, no se reconoce la estética propia de la tecnología digital y en cambio se cree descubrir en ella procesos nuevos, cuando estos son ya patrimonio de tradiciones artísticas consagradas históricamente. Al respecto, el ejemplo típico es la multimedia, que existe desde la prehistoria (la danza, el teatro...); pero este caso no es el único pertinente, se podría decir lo mismo de la intersección entre ciencia, matemática y arte (véase la decoración árabe, la sección áurea, la perspectiva, etcétera); por último, también lo que más parece novedoso de lo digital, los procesos automatizados y algorítmicos tienen raíces lejanas (véase Ramón Lull y su Ars Magna) y de todas maneras mucho más antiguas que las computadoras. Por ejemplo, Hermann Hesse escribía en 1930:

... el borrador de una historia de El juego de abalorios, que pasando desde los seminarios musicales a los de matemática (...) había llegado a un nivel de sofisticación tal que podía expresar procesos matemáticos con signos y especiales abreviaciones; los jugadores se intercambiaban dichas fórmulas abstractas desarrollándolas recíprocamente y se presentaban uno con el otro los desarrollos y las posibilidades de sus ciencias. $^{2}$

1 ARNS, Inke. "Read_me, run_me, execute_me: Some notes about software art" [en línea]. Lecture at Kuda, Novi Sad, 9 de abril del 2004. <http://www.v2.nl/ arns>.

2 HESSE, Hermann. Das Glassperlenspiel. Zurich: Fretz und Wasmuth Verlag, 1943. (Traducción al italiano: Il gioco delle perle di vetro. Milán: Mondadori, 1955, p. 29; traducción al español: El juego de aba- 
$\mathrm{Y}$ esto fue imaginado incluso antes de As We May Think, el célebre ensayo de Vannevar Bush, ${ }^{3}$ que se considera el manifiesto de los hipertextos y del cyberespacio.

Por todas estas razones, el lenguaje de la tecnología digital se percibe casi exclusivamente por medio del espectáculo de sus aparatos audiovisuales y no produce obras estéticamente significativas; en suma, los hipertextos y la multimedia no han superado el estadio de la experimentación literaria, y el arte digital casi no interesa fuera de un pequeño círculo de nerds.

Pero sería un error pensar que las posibilidades estéticas del digital se acaben en la banalidad de los efectos especiales o en la élite del tecnoarte. Me parece que el horizonte estético que caracteriza estructuralmente lo digital, si existe uno, es el contexto de la abertura y de la indeterminación, temas modernistas sobre los que Eco ha teorizado en los años sesenta en su Obra abierta. Dicha estética apela, de modo evidente, a los procesos interactivos y multimediales típicos del digital, que sin embargo ha introducido nuevos y contradictorios elementos en las estructuras indeterminadas y abiertas de la literatu- ra o de la música examinadas en el trabajo de Eco. Uno es el cuestionamiento de la autoría - implícito en la estética de la obra abierta - planteado por el digital en una forma mucho más radical, tanto más radical que se llegaría a discutir no solo la muerte del autor (la creación), sino también la muerte del lector (la interpretación).

Sucede que las ambigüedades que he señalado anteriormente hacen que cuando se desarrolle una estética de la interacción multimedia, este desarrollo pertenezca mucho más al momento de la creación del artista que al proceso de interpretación y al papel creativo del lector, pues este todavía recibe las obras interactivas y multimediales, según el tradicional paradigma secuencial y cerrado del cine o de la televisión. Una interactividad y una abertura que son simplemente un maquillaje que esconde procesos estéticos obsoletos.

Por esto, hay que definir en primer lugar el significado de interacción. Ahora bien, este significado no se reduce al control de algún software por medio de alguna interfaz, porque la interacción se debería entender en el sentido intercultural. Digo esto por la naturaleza misma de la computadora: esta, valga la

lorios: ensayo de una biografía del "magister ludi" Josef Knecht, junto con los escritos dejados por él. Buenos Aires: Santiago Rueda, 1949).

3 BUSH, Vannevar. "As We May Think". Athlantic Monthly, 1945. Versión electrónica de Denys Duchier, abril de 1994. dduchier@csi.uottawa.ca. 
repetición, no es solo una máquina sino un sistema culturalmente complejo; utilizando la computadora, la interacción implica entonces no solo una comunicación de mensajes performativos, sino el intercambio de intenciones, finalidades, experiencias y la distribución de conocimientos. El filósofo y crítico mediático McKenzie Wark señala que:

La información quiere ser libre, pero está por todos lados encadenada. (...) La libre info.mación debe ser libre en todos sus aspectos, como archivo, como proceso, como medio de distribución y de comunicación. ${ }^{4}$

Pero la computadora como sistema no garantiza esta condición, por el contrario, es un sistema opaco, cuyos procesos el usuario percibe en modo muy limitado y poco transparente. Y según Inke Arns:

En el lenguaje común transparencia significa normalmente visibilidad o claridad, o control a través de la visibilidad. Sin embargo transparencia en el caso de la computación es sinónimo de ocultamiento de información, que significa que el usuario no se percata de los procesos internos del software. 5

Así es que si no existe transparencia la interacción se da en un solo sentido, que va desde el elemento más fuerte (el sistema tecnológico) al elemento más débil (el usuario) y se resuelve en alguna forma de persuasión, de manipulación y de ejercicio de poder.

Dicho esto podemos pasar a examinar la naturaleza de la multimedia digital, pues el sentido más amplio de interacción que estamos discutiendo se manifiesta de manera clara y con aspectos peculiares precisamente dentro esta tecnología. Quizás lo más interesante consiste en que en el contexto de la esencia numéricamente abstracta del digital la multimedia no significa tanto el uso de varios medios sino más bien la interacción entre ellos. Sucede que el proceso de digitalización equipara los lenguajes y los procesos específicos de los medios analógicos en el espacio homogéneo de ceros y unos. Esto implica que, por primera vez, los medios artísticos comparten un lenguaje común y pueden dialogar e intercambiarse datos, procesos y conocimientos. Es por esta razón, es decir, porque manipulamos números y no medios, que podemos interpretar los datos que codifican una imagen como si fueran los que representan una partitura musical y viceversa: así mismo, podemos aplicar procesos creativos típicos de la música (composición, armonía, contrapunto, etcétera) a la generación de una imagen, de una arquitectura o de un texto.

4 MCKENZIE, Wark. A Hacker Manifesto. Cambridge: Harvard University Press, 2004, pp. 126 y 138.

5 ARNS, Inke. Op. cit. 
Este lenguaje común es, evidentemente, el lenguaje lógico matemático, que amplifica la portada de estas dinámicas creativas en un modo todavía no bien conocido, porque agrega también las posibilidades y los procesos de las nuevas ciencias de la complejidad. En efecto, por medio de la simulación los procesos biológicos, físicos o químicos se convierten en medios creativos y se descubre en términos metódicos la belleza emergente de las formas naturales (los fractales, la vida artificial, el arte orgánico, para nombrar algunas técnicas).

La estética multimedia consiste, quizás contrariamente al sentido que por lo común se le otorga, no tanto en las facilidades hardware y software para coordinar operativamente video, gráfica y música en un mismo entorno automatizado, sino en el reto de dominar estos diferentes lenguajes expresivos en un contexto interdisciplinario y epistemológicamente contradictorio (a pesar de algunas teorías posmodernas que tienden a anular, así no más, las diferencias entre ciencia, arte y filosofía).

Desde luego, podemos empezar a hablar de hipermedia, es decir, de hipertextos multimediales y de procesos interdisciplinarios (que se originan de la relación con el entorno sistémico de la ciencia). Pero así se plantea con toda evidencia la cuestión del monopolio del conocimiento y del know how, pues, en la práctica, las herramientas multimedia amplifican este problema porque sus algoritmos se hacen cargo, en lugar del usuario, de dominar las dificultades técnicas de cada medio; así se puede creer que se es creador, cuando en realidad se está utilizando el know how incrustado en el código del software. Por otro lado, este know how no es compartido, es patrimonio privado del software que las interfaces se encargan de ocultar (bajo el pretexto de facilitarle el trabajo al usuario). Más aún, las interfaces se convierten en un medio que utiliza y condiciona al usuario, porque este, sin darse cuenta, se encuentra operando dentro de un contexto altamente mediado y sin que los términos de esta mediación sean explicitados. Paradójicamente - aunque no tanto- las interfaces son más funcionales a los objetivos del software (de quienes lo producen y lo venden) que sensibles a nuestras exigencias creativas.

Podemos decir, entonces, que la interacción digital (en su sentido cultural) es un proceso que está contaminado: el artista se vuelve fácilmente un apéndice del software y, por lo tanto, su obra es estéticamente inconsistente. Es por esto que los artistas digitales serios son siempre programadores (producen su propia tecnología), porque así pueden gestionar el proceso creativo e interactivo sin interferencias y mediaciones indeseadas. Pero esta es una tarea complicada, si se quiere generalizar el modelo, porque aumenta el nivel de com- 
petencias que se necesita para llevar a cabo un proceso creativo independiente. Ahora bien, aun suponiendo que este proceso de transformación del artista - algunos hablan de neorrenacimiento- tenga éxito, queda sin embargo irresuelto el problema desde el lado del lector: a menos que sea otro artistaprogramador, este no necesariamente domina todos los conocimientos que se requieren para la interpretación y debería contar con alguna mediación o interfaz interpretativa. En efecto, para la estética abierta las metáforas visuales de las interfaces y las obras de arte son filtros que interfieren en el acceso a la información y a la libertad interpretativa del lector de la cual esta información depende.

En el contexto de la interacción como valor estético del arte digital se plantean dos problemas estructurales: primero, los medios interactivos no son realmente tales; por el contrario, son aparatos que permiten una sutil manipulación ideológica y cultural; luego, la experiencia de la apertura y de la creación interactiva, que el digital garantiza como hardware, implica un proceso software de intercambio de conocimientos, que en realidad no sucede. Precisamente porque software e interfaces son algún tipo de relato estético que interfiere con la creación interactiva, se genera una contradicción inesperada entre la tecnología y la estética de la obra abierta.
Una primera tarea para lograr una estética de la interacción digital requiere, entonces, asumir, después de Eco, el significado radical de apertura: esta ya no consiste en un cierto grado de libertad interpretativa dentro un contexto estructurado, sino en la libre construcción de estéticas individuales dentro del espacio interactivo de la obra de arte; ahora, la función del arte es puramente medial, porque procesa los contextos del artista y del espectador (si todavía tiene sentido utilizar estos términos) y sus respectivos conocimientos con el horizonte social, histórico y cultural. En efecto, la obra de arte abierta digital desaparece totalmente como entidad definida y se convierte en un instrumento, porque sus resultados emergen libremente desde el contexto sistémico de la interacción.

Consecuentemente, un segundo reto consiste en reubicar la identidad y la calidad (el aura en el sentido que le dio Benjamin) de la obra de arte y del artista. Ahora bien, la calidad se encontrará nuevamente en el diseño del desarrollo interactivo y de la arquitectura hermenéutica y mayéutica del proceso artístico; pero este aura no es el producto arbitrario del genio individual, que Benjamin tenía como blanco de su crítica, sino que posee un valor epistemológico porque se fundamenta en el intercambio de conocimiento. Y hoy, gracias también al digital, este conocimiento histórico, estético y social 
incluye y se armoniza de una forma original con las nuevas investigaciones de las ciencias metódicas.

Para concluir, los procesos abiertos e interactivos dependen, sin embargo, de las estructuras del software y de las interfaces, en cuanto son mediadores de la experiencia y el conocimiento. Son estas las que, en primer lugar, habría que comenzar a estudiar en sus componentes filosóficos y epistemológicos si se quiere fundar una estética digital que no se limite a reproponer modelos existentes que, además, ya están cuestionados por la historia y la sociedad. En efecto, hoy software e interfaces son aparatos por los cuales el digi- tal, paradójicamente, no solamente no construye una estética propia, sino que actúa como vector de instancias obsoletas, generando contradicciones evidentes con aquel pensamiento posmoderno que algunos han visto reflejado en la aparente fragmentación y multiplicidad del cyberespacio.

La importancia de la experimentación llevada a cabo por los artistas digitales juega aquí un papel sociocultural importante, ya que es la única forma con la que se puede deconstruir y reconstruir libremente, fuera de la lógica del mercado o del ejercicio del poder, los predominantes procesos de la tecnociencia y de la globalización cultural. 